\title{
Diffractive Design
}

\section{Corinna Bath}

Gender, Technik und Mobilität, Institut für Flugführung, Technische Univeristät Braunschweig

Ostfalia Hochschule für angewandte Wissenschaften

\section{Einleitung}

Bislang konzentriert sich die Geschlechter-Technik-Forschung auf Frauen und ihren unzureichenden Zugang zu technischen Studiengängen und Berufen (Leicht-Scholten, 2007; Koreuber, 2010; Quaiser-Pohl und Endepohls-Ulpe, 2010; Stöger, Ziegler und Heilemann, 2012). Wenige Untersuchungen nehmen ingenieurwissenschaftliche Fachkulturen und Curricula oder die Nutzung von Technik in den Blick (Bauer und Götschel, 2006; Maass und Wiesner, 2006). Noch geringer sind Erkenntnisse über problematische Vergeschlechtlichungen von Inhalten und Methoden technischer Studiengänge und von technischen Produkten (vgl. Zorn, Maass, Rommes, Schirmer und Schelhow, 2007; Sørensen, Faulkner und Rommes, 2012). Mit Projekten wie „Gendered Innovations“ (vgl. http://genderedinnovations. stanford.edu/) wurde zwar der Versuch unternommen, die Bedeutung der Kategorie Geschlecht für die natur- und ingenieurwissenschaftliche Forschung und Entwicklung zu verdeutlichen. Allerdings wird dort mit einem Geschlechterverständnis von „sex“ und „gender“ gearbeitet, das an den meisten Orten der Geschlechterforschung als überholt gilt (vgl. Butler, 1991; Becker-Schmidt und Knapp, 2000; Degele, 2008). So wird etwa von der Möglichkeit einer analytischen Trennung von ,sex“ und ,gender“ ausgegangen, ohne auf die für die Geschlechterforschung grundlegende Sex-Gender-Debatte Bezug zu nehmen, die die Unterscheidbarkeit der beiden Begriffe prinzipiell mit dem Argument in Frage gestellt hat, dass es kein vorsoziales, unhistorisches, nichtkulturelles Körperliches geben kann. Ferner wird, statt zu untersuchen, wie Zweigeschlechtlichkeit und Heteronormativität sozio-materiell hergestellt werden, vielfach gesetzt, was Gegenstand der Analyse sein sollte (vgl. Bath und Both, 2014; siehe auch Bath, 2007).

In diesem Beitrag möchte ich Diffractive Design als eine Designphilosophie zur Technikgestaltung in der Informatik vorschlagen, die darauf zielt, die genannten Desiderate zu überwinden. Im Folgenden geht es somit um die Frage, wie sich Geschlechterforschung und Technikgestaltung in der Informatik produktiv miteinander ins Gespräch bringen lassen. Dazu greife ich auf die physikalische Metapher der Diffraktion bzw. Interferenz zurück, die von der Physikerin und feministischen Wissenschaftstheoretikerin Karen Barad im Anschluss an Donna Haraway in Diskussion gebracht worden ist.

Im folgenden Abschnitt erläutere ich Barads Verständnis von Diffraktion, um im nachfolgenden Abschnitt die Bedeutung der drei Ebenen des Begriffs - optische Metapher, Epistem- 
onto-logie und Relationalität mit dem Unerfassbaren und den Anderen - für die Informatik zu diskutieren. Der nächste Abschnitt führt davon ausgehend in kritische Technikgestaltungsansätze ein, die gesellschafts- und wissenschaftstheoretische Erkenntnisse in informatische Methoden integriert haben.

Diffractive Design baut auf solch vielversprechenden vorliegenden Ansätzen auf. Zurückgegriffen wird dabei ebenso auf Ansätze, die Geschlechteranalysen in Technikentwicklung übersetzen und beispielsweise der Frage nachgehen: Wie lässt sich Technik so gestalten, dass die problematischen Vergeschlechtlichungen von Software, IT und ihren Grundlagen, die in vorliegenden Forschungen bereits identifiziert worden sind, vermieden werden? Diffractive Design führt diese unterschiedlichen Ansätze interferent im Sinne eines „Durch-einanderhindurch-Lesens“ mit dem Ziel guter Technikgestaltung zusammen, so dass am Ende „lebbaren Welten“ im Sinne Donna Haraways und „lebenswerte Leben“ im Sinne Judith Butlers entstehen können.

Interferenz bzw. Diffraktion ${ }^{1}$ bezeichnet das physikalische Phänomen der Überlagerung von Wellen, die sich an bestimmten Stellen verstärken oder aufheben. Es bilden sich Interferenzmuster, in denen Unerwartetes sichtbar und scheinbar Selbstverständliches verschwinden kann. Die feministische Wissenschafts- und Technikforscherin Karen Barad (2007, 2012, 2013) hat diesen Begriff im Anschluss an Donna Haraway (1995) aufgegriffen, um eine Prozesshaftigkeit von Seins- und Wissensformen zu denken, die weder nach Logiken der Ausschließung oder Ermächtigung funktioniert, noch Vorstellungen fester Identitäten bedient. Barad nutzt damit eine Metapher, in die aktuelle Ansätze der Geschlechterforschung bereits eingelassen sind. Denn Geschlechterforschung begreift Frauen und Männer, Weiblichkeit und Männlichkeit nicht als etwas Feststehendes, das Identität bestimmt. Vielmehr wird Geschlecht als ein Prozess verstanden. Wir aktualisieren Geschlecht ständig in unseren alltäglichen Interaktionen. Wir stellen Geschlecht als Norm ständig wieder neu her. Dabei scheint wie bei der Überlagerung von Wellen weder ein eindeutiger Bezug auf ein Original möglich zu sein, noch entsteht am Ende das Immer-Gleiche. Es kommt vielmehr in jeder Iteration zu Verschiebungen und Veränderungen. Auch deshalb lassen sich Ausschluss oder Ermächtigung nicht einfach kausal und für eine Gruppe denken, die damit ungerechtfertigt als homogen angenommen wird.

Gleichzeitig bietet der Rückgriff auf die Physik die Möglichkeit einer Übersetzung zwischen MINT-Fächern und Gender Studies bzw. den Sozial- und Geisteswissenschaften. Über das optische Bild der Interferenz hinausgehend, verknüpft Barad den Begriff zugleich mit quantentheoretisch-philosophischen Überlegungen, die der Physiker Nils Bohr in Diskussion gebracht hatte. Paradigmatisch steht dafür das Doppelspaltexperiment, das je nach Versuchsaufbau (wird „gemessen“, welche Teilchen durch den einen oder anderen Spalt hindurch gehen, oder wird nicht ,gemessen“) die für Wellen typischen Interferenzmuster oder die für Materie typischen Muster als Ergebnis zeigt. Dies lässt sich zunächst als Einflussnahme der

\footnotetext{
${ }^{1}$ Ich folge Barad (2007) darin, die Begriffe Diffraktion und Interferenz synonym zu verwenden, um auf die enge Verknüpfung von Wissen und Sein sowie die Problematik von Kausalität hinzuweisen, obwohl Diffraktion im physikalischen Sinne meist als Beugung (also in Bezug auf den Prozess der Entstehung und Herstellung von Interferenzen) verstanden wird, während Interferenzmuster den Effekt von Beugung bezeichnen.
} 
Forschenden auf das Ergebnis der Forschung lesen - ein Phänomen, das in den selbstreflexiven Sozial- und Geisteswissenschaften geläufig ist, wird damit auch physikalisch belegt.

Die Paradoxie, dass Materie unter bestimmten Umständen auch Eigenschaften von Wellen aufweisen kann, ist damit jedoch nicht aufgelöst oder erklärt. Während Heisenberg diese Paradoxie als Unschärfe - und damit als ein epistemologisches Problem - begreift, versteht sein Lehrer Bohr es als ein ontologisches: Die Teilchen „haben“ Wellen- oder Materiecharakter, sie „haben“ bei der Messung im Beschleuniger entweder Ort oder Impuls, aber nicht beides gleichzeitig. Dies deutet darauf hin, dass Wissen und Sein noch viel enger miteinander verknüpft sind als gemeinhin angenommen. Barad spricht deshalb von auch von Epistemonto-logie oder Onto-epistemo-logie.

Barads bzw. Bohrs Denken geht darüber hinaus. Sie behaupten mit Hilfe weiterer quantenphysikalischer Experimente, dass Sein und Wissen keine individuellen und objektivistischen Projekte, sondern radikal relational sind. Diese Relationalität sei zudem grundlegend durch Andere und Anderes bestimmt, die immer nur teilweise begreifbar und beschreibbar sein können.

Ein solches Verständnis könnte lähmen, handlungsunfähig machen. Es kann jedoch auch zu neuen Denkweisen inspirieren. Es war Haraway, die mit Bezug auf Trinh Min-ha bereits in ihren frühen Schriften auf das politische Potential des Begriffs „un/an/geeignete Andere“ (Haraway, 1995, S. 20-21) aufmerksam gemacht hat, der in diesem Zusammenhang hilfreich erscheint, um diese physikalischen Phänomene mit Erkenntnissen der Gender und Postcolonial Studies in Beziehung zu setzen. „Un/an/geeignete Andere“ bringen nicht nur das Verworfene, die jenseits der Norm Stehenden, die/das nicht wissbare(n) Andere(n) mit ins Spiel, sondern ermöglichten ,die Differenzbeziehungen zwischen den Völkern und zwischen menschlichen Wesen, anderen Organismen und Maschinen neu fassen [...]: nicht als hierarchische Herrschaft, Einverleibung von Teilen in Ganzheiten, paternalistische und kolonialistische Protektion, symbiotische Verschmelzung, antagonistische Opposition oder instrumentelle Produktion aus der Ressource.“ (ebd.). Übliche Gesten der Unterwerfung ließen sich damit ebenso vermeiden wie ein naiver Versuch des Ausbrechens aus bestehenden Herrschaftskonstellationen. Es sei jedoch, wie Haraway betont, eine „harte intellektuelle, kulturelle und politische Arbeit" erforderlich, um eine solche bessere Welt zu denken und zu produzieren. „[E]ine differentielle, [interferente] feministische Allegorie [könnte] die „un/an/geeigneten Anderen“ [...] in eine Science-Fiction-Welt mit Namen Anderswo entlassen. Das wäre der Ort, der sich aus [Interferenzmustern] zusammensetzte.“ (ebd.). Es sind demnach also gerade Diffraktionen/Interferenzen, die denjenigen und dasjenige, die/das bisher außerhalb der Norm und/oder außerhalb des Wissens stehen, ein Sein und lebenswertes Leben eröffnen.

\section{3 \\ Diffraktive Verknüpfungen von Informatik und Geschlechterforschung}

Die drei skizzierten Ebenen von Interferenz bzw. Diffraktion - optische Metapher, Epistemonto-logie und Relationalität mit dem Unerfassbaren - erlauben es, Verknüpfungen von Informatik und Geschlechterforschung neu zu denken. Als optische Metapher stellt Diffraktion meines Erachtens ein besseres Leitbild für interdisziplinäres Arbeiten dar als die bisher zumeist praktizierten Modelle, bei denen eine der Disziplinen mit ihren Theorien, Methoden 
und Arbeitsweisen letztendlich als Maßstab der Beurteilung gesetzt wird. Barad (2013, S. 60 ff.) wendet sich explizit gegen solche unidirektionalen Modelle, die beispielsweise versuchen mit physikalischen oder biologischen Konzepten gesellschaftliche Entwicklungen zu erklären oder auch umgekehrt die Gesellschaft als Modell für die Naturwissenschaften heranzuziehen. Beim „Durch-einander-hindurch-Lesen“ - wie Barad ihren methodischen Ansatz auch bezeichnet - sollen zwei oder mehrere Theorien verschiedener disziplinärer Herkunft gleichwertig behandelt werden, wobei auch kleinste Unterschiede berücksichtigt werden sollen. Ihres Erachtens reicht es jedoch nicht, Zweibahnstraßen zu bauen und ,die Ergebnisse dessen zu addieren, was passiert, wenn jede der Theorien mal an der Reihe ist, das Gegenstück zu spielen“ (ebd.).

Barads Ziel ist es vielmehr, die aus den verschiedenen disziplinären Praktiken hervorgehenden Verstehensweisen ,auf Augenhöhe“ miteinander ins Gespräch zu bringen. So wie zwei oder mehrere Wellen, die aufeinander treffen, nicht gleich bleiben, sondern sich überlagern, an manchen Stellen verstärken, an anderen nivellieren, gehen die beteiligten disziplinären Ansätze aus einem solchen Prozess radikal interdisziplinärer Konversation nicht als dieselben hervor. Sie sind im Effekt so miteinander verknüpft, dass sie Interferenzmuster bilden. Dabei sind - wie die physikalischen Experimente zeigen - bereits kleinste Differenzen entscheidend für das, was am Ende als Ergebnis entsteht. Deshalb legt Barads diffraktive Methodologie höchste Aufmerksamkeit auf Unterschiede: „Diffraktion ist auch eine geeignete Metapher zur Beschreibung meines methodologischen Ansatzes, Einsichten durch einander hindurch zu lesen und dabei die Details und Besonderheiten von Differenzbeziehungen und deren Auswirkungen zu beachten und auf sie einzugehen." (Barad, 2013, S. 28).

Bis diese Vision umgesetzt sein wird und wir zu einem solch interdisziplinären Arbeiten zwischen Informatik und Geschlechterforschung gekommen sind, ist es vermutlich noch ein langer Weg. Nichtsdestotrotz erscheint mir die optische Metapher der Diffraktion, ausbuchstabiert als ein „Durch-einander-hindurch-Lesen“, als ein vielversprechender Ansatz, um informatische Arbeitsweisen für die Geschlechtertheorie produktiv zu machen und Erkenntnisse der Geschlechterforschung in informatische Produkte umzusetzen.

Auch der zweite Aspekt des Diffraktionskonzepts, die Verknüpfung von epistemologischen und ontologischen Aspekten, erscheint mir für die Verknüpfung von Informatik und Geschlechterforschung äußerst relevant. Kaum eine andere technisch-orientierte Disziplin verbindet das materielle Gestalten von Welt, d. h. von Sein, so stark mit der informationellen Gestaltung, d. h. der Gestaltung der Grundlagen von Wissen, wie die Informatik. Als Wissenschaft der Informationsverarbeitung besteht eine ihrer Kernbeschäftigungen in der Modellierung und automatischen Bearbeitung von Wissen. Informatische Konzepte und Abläufe beeinflussen zunehmend, was wir denken können, und vor allem das, was undenkbar ist und verworfen wird oder nicht sein darf. Dirk Siefkes und die Kolleg_innen des Projekts zur Sozialgeschichte der Informatik betonten, dass die Gegenstände der Informatik „Hybridobjekte" seien: einerseits als Notationen immateriell-geistig, andererseits wird ihnen eine spezifische Form der Wirkmacht, der Bewegung und des Agierens zugeschrieben, die materiell wirksam ist. Sie sind gleichzeitig Zeichen und Gegenstand (Siefkes, Eulenhöfer, Stach und Städtler, 1998, S. 3-4). Sie werfen damit sowohl wissenschafts- und erkenntnistheoretische Probleme (Epistemologie) als auch ontologische Probleme auf. Interferenz ermöglicht, diese aus einer geschlechterkritischen Perspektive zu bearbeiten und Fragen der Verantwortung als Wissenschaftler_in bzw. Informatiker_in zu diskutieren (vgl. Bath, 2013, Semantic Web). 
Interferenz verweist auf die Notwendigkeit, uns mit unserem - teils unvermeidbaren - Eingreifen auseinanderzusetzen, selbst wenn wir die Folgen dieser Eingriffe nicht vollständig wissen können. Wenn wir Barads Ansatz folgen, kann Wissen nicht Repräsentation von Welt sein, sondern bedeutet ihr machtdurchzogenes Schaffen. „Interferenzen” fordern somit eine ethisch, erkenntnistheoretisch und politisch fundierte Praxis - auch in der Technikgestaltung - ein.

Damit stellt sich nicht nur die Frage nach der Technikgestaltung, sondern auch nach der Wissensproduktion im Angesicht unvermeidlicher Alterität: Wie lassen sich Technologien konstruieren, wenn die Welt draußen, wir selbst, unsere Apparate und ihre intra-relationalen Verwicklungen stets in Teilen unklar bleiben müssen, wenn wir sie nicht vollständig wissen können? Diese Frage kann zugleich politisch gewendet und aus einer geschlechtertheoretisch-feministischen Perspektive untersucht werden: Wie kann durch eine geeignete Modellierung von Welt, durch eine geeignete Konstruktion von Artefakten und Algorithmen und durch passende epistem-onto-logische Annahmen ein Ort für „un/an/geeignete Andere“ geschaffen werden?

In den folgenden Abschnitten wird diskutiert, wie dieser Anspruch in Technikgestaltung in der Informatik übersetzt werden kann.

\section{4}

\section{Kritische Technikgestaltungsansätze}

In der kritischen Informatik gibt es eine Reihe von Ansätzen, die Technikgestaltung mit Gesellschafts- und Wissenschaftskritik, Ökonomiekritik und Ethik verknüpfen. Am bekanntesten ist darunter das Participatory Design (PD) der skandinavischen Schule (vgl. Greenbaum und Kyng, 1991; Kuhn und Muller, 1993; Bødker et al., 2004), bei dem zukünftige Nutzer_innen möglichst früh und möglichst gleichberechtigt an der Entwicklung von Technik beteiligt werden sollen. Es umfasst ein breites Methodenspektrum, das auf den regelmäßig stattfindenden PDC-Konferenzen diskutiert wird.

Participatory Design der skandinavischen Schule steht in marxistischer Tradition und grenzte sich früh gegen sozio-technische Ansätze ab, die als zu management-orientiert betrachtet wurden. Demgegenüber verstand sich Participatory Design stets als eine politisch und gewerkschaftlich motivierte, technologische Forschung und Entwicklung für diejenigen, die gesellschaftlich strukturell benachteiligt sind. Es entstanden seit den 1990er Jahren auch viele Projekte von Frauen für Frauen, insbesondere für Beschäftigte in typischen Frauenberufen (vgl. insbesondere Hammel, 2003). Kritik an der Partizipationsforschung wird primär daran geübt, dass die politischen Ansprüche angesichts von Modernisierungs- und Neoliberalisierungsstrategien aufgegeben oder nicht mehr eingelöst werden können.

Einen zweiten für diesen Beitrag relevanten kritischen Technikgestaltungsansatz stellt das Reflective Design (Sengers, Boehner, David und Kaye, 2005) dar, das ich hier insbesondere auch deshalb diskutieren möchte, weil Barad Diffraktion bzw. Interferenz der Reflektion gegenüber stellt. Reflective Design zielt primär darauf, kulturell verankerte Werte und nicht reflektierte Annahmen, die technischen Artefakten bzw. den Methoden und Praktiken zu ihrer Konstruktion eingeschrieben werden, bewusst zu machen, um auf dieser Grundlage alternative Gestaltungsmöglichkeiten zu entwickeln und auszuprobieren. Die zu konstruierenden Artefakte sollen den Nutzer_innen nicht nur umfassende Erfahrungen, sondern zugleich eine 
Reflektion ihrer gesellschaftlich-kulturellen Selbstverständnisse ermöglichen: „,[R]eflection itself should be a core technology design outcome of HCI" (Sengers et al., 2005, S. 50).

Dazu wird „Reflektion“, d. h. gesellschaftskritische bzw. -theoretische Ansätze, mit Technikgestaltung kombiniert. Reflective Design nimmt theoretische Anleihen bei marxistischen, feministischen und postkolonialen Ansätzen, den Kultur- und Medienwissenschaften sowie der Psychoanalyse. Auf dieser Grundlage sollen bei der Technikgestaltung relevante Selbstverständnisse offen gelegt und somit - als ein erster Schritt möglicher Veränderung - der bewussten Entscheidung zugänglich gemacht werden. „Critical theory argues that our everyday values, practices, perspectives, and sense of agency and self are strongly shaped by forces and agendas of which we are normally unaware such as the politics of race, gender and economics" (ebd.).

Damit bietet der Ansatz prinzipiell die Möglichkeit, Vergeschlechtlichungen technischer Produkte zu begegnen, die aus impliziten problematischen Geschlechtervorstellungen resultieren. Insbesondere aufgrund seines Bezugs auf feministische und Geschlechtertheorien birgt Reflective Design das Potential, Festschreibungen, Stereotypen und vorherrschenden gesellschaftlichen Selbstverständnissen von (Zwei-)Geschlechtlichkeit und Heteronormativität entgegenzuwirken.

Um diese gesellschafts- und geschlechtertheoretischen Ansprüche in den Prozess und die Praxis der Technikgestaltung umzusetzen, arbeitet Reflective Design mit verschiedenen methodischen Ansätzen, die je nach Problemlage ausgewählt und miteinander kombiniert werden können. Zum Einsatz kommen Elemente aus dem Participatory Design, dem Value Sensitive Design, Critical Technical Practice sowie dem Design for Experience. Ferner gründet Reflective Design im Critical Design, Ludic Design und dem Reflection-in-Action-Ansatz (vgl. Bath, 2009, für eine Beschreibung und geschlechterwissenschaftliche Einordnung dieser Ansätze). Ziel ist es, dass sowohl der Prozess der Gestaltung als auch das technische Produkt Reflektionen bei den Designer_innen und Nutzer_innen evoziert. Mithin geht es darum, die technischen Produkte selbst als Akteur_innen kritischer Positionen zu konzipieren.

Im Vergleich zu den meisten anderen Methoden kritischer Technikgestaltung zielt Reflective Design jedoch nicht nur auf die Nutzung, sondern setzt zugleich bei den Technikgestalter_innen an. Dies korrespondiert mit der Verschiebung, nicht allein auf Partizipation und Erfahrung zu fokussieren, sondern auch darauf, Voraussetzungen und grundlegende Annahmen bei der Gestaltung von technischen Artefakten herauszuarbeiten und gegebenenfalls zu verändern. Ferner ist es mit diesem Ansatz möglich, über die Gestaltung von Software und Informationstechnologien hinaus zu gehen und auch Grundlagen und Voraussetzungen dieser in den Blick zu nehmen. Es ist insofern möglich, epistemologische (bzw. epistem-ontologische) Annahmen und Grundlagen der Technikgestaltung, etwa Modellierungsansätze oder Algorithmen, kritisch wie feministisch zu problematisieren und gegebenenfalls neu zu gestalten.

\section{5}

\section{Diffractive Design}

Barad und Haraway ernst nehmend, genügt eine solche Reflektion in der Technikgestaltung, wie sie das Reflective Design vorschlägt, nicht. Es geht vielmehr darum, über die Repräsen- 
tationsansätze, von denen die Informatik geprägt ist, die sich auch in zahlreichen kritischen sozial- und geisteswissenschaftlichen Ansätzen finden, hinauszugehen.

„Diffraktionsmuster zeichnen die Geschichte von Interaktion, Interferenz, Verstärkung und Differenz auf. Diffraktion handelt von heterogener Geschichte, nicht von Originalen. Anders als Reflexionen verschieben Diffraktionen nicht das Gleiche in mehr oder weniger verzerrter Form woanders hin. ... Eher kann Diffraktion als eine Metapher für eine Art kritischen Bewußtseins am Ende dieses ziemlich schmerzhaften Christlichen Milleniums sein; eine, die sich verschreibt, einen Unterschied zu machen anstatt das Heilige Ebenbild zu wiederholen." (Haraway, 1997, S. 273, zitiert in der Übersetzung nach Barad, 2013)

Um nicht im Ewiggleichen befangen zu bleiben und insbesondere die bestehende strukturellsymbolische Geschlechterordnung überwinden zu können, bietet sich Diffraktion somit als ein besserer Begriff als Reflektion an. Eine weitere Herausforderung liegt darin, das Unerfassbare und die un/an/geeigneten Anderen, die sowohl im Eigenen wie im Außen liegen können, mit in die Konzeption, Modellierung und Gestaltung technischer Artefakte einzubeziehen. Denn gerade die Informatik rekonfiguriert mit ihren Artefakten zunehmend, was wir wissen und denken können, wie wir handeln und damit auch, was wir sind..

Diffractive Design ist die Vision einer kritischen Technikgestaltungsmethode, die darauf zielt, genau diese Aufgabe zu erfüllen. Dazu werden auf Basis der von Barad vorgeschlagenen Methode die zu konzipierenden technischen Artefakte mit kritischen Technikgestaltungsmethoden und dem Interferenzansatz durch-einander-hindurch-gelesen. Diffractive Design kann methodisch auf den Ansätzen des Reflective Design und des Participatory Design aufbauen. Welche kritischen Technikgestaltungsansätze genau zum Einsatz kommen sollen, ist spezifisch zu prüfen und sollte auf einer fundierten Analyse beruhen.

Eine Möglichkeit besteht darin, nach der von mir vorgeschlagenen Vorgehensweise des „DeGendering informatischer Artefakte“ (Bath, 2009; Bath, 2014) vorzugehen. Dabei wird von der Analyse problematischer Vergeschlechtlichungen informatischer Artefakte ausgegangen, für die ich vier Dimensionen differenziert hatte: 1 . strukturell bedingte Ausschlüsse bestimmter Nutzer_innengruppen von der Technologie, die häufig durch die so genannte „I-Methodology“ (Akrich, 1995) in der Technikgestaltung zustande kommen, 2. Ein- und Festschreibungen der vorherrschenden geschlechterhierarchischen Arbeitsteilung, die oft auf unangemessenen Annahmen über weibliche und männliche Kompetenzen oder die mangelnde Berücksichtigung der Geschlechterpolitiken im Anwendungsfeld zurückzuführen sind, 3. die Normalisierung von Geschlecht durch die explizite Repräsentationen von geschlechtlichen Körpern und Verhaltensweisen in Artefakten und 4. Geschlechterpolitik und Epistemologie der Modellierung und Grundlagenforschung in der Informatik, die mit De-Kontextualisierung, traditionellen Objektivitätsauffassungen und $\mathrm{zu}$ hinterfragenden ontologischen Annahmen verwickelt sind. Diesen Problematiken wurden jeweils angepasste Methoden der Technikgestaltung, die auf ein De-Gendering zielen, entgegengestellt. Dabei hatte ich über Ansätze des User-Centred Design, des Participatory Design und Reflective Design hinaus gehend je nach Kontext und Problem weitere Methoden der kritischen Technikgestaltung vorgeschlagen wie das Underdetermined Design (Cassell, 2003), Mind Scripting (Allhutter, Hanappi-Egger und John, 2008; Allhutter, 2012), Value Sensitive Design (Friedman und Kahn, 2003; Flanagan, Nissenbaum, Diamond und Belman, 2007) oder Critical Technical Practice (Agre, 1997). Neben der von der Geschlechterforschung inspirierten Untersuchung problematischer Vergeschlechtlichungen von Artefakten können auch - wie 
beim Research and Development (GERD) (Wajda, Draude, Maass und Schirmer, 2013) Phasen der Software-Entwicklung zum Ausgangspunkt der Analyse gemacht werden oder auf andere Verknüpfungen von Analyse und Design (vgl. etwa Wagner, Brattetei und Stuehldahl, 2010; Rommes, Bath und Maass, 2012) zurückgegriffen werden.

Diffractive Design gibt hier kein Vorgehen vor, sondern verlangt vielmehr eine differenzierte Analyse des Ausgangsproblems dessen, was sozio-technisch rekonfiguriert werden soll (vgl. Suchman, 2007). Es wären weitere methodische Elemente zu ergänzen, welche zusätzlichen Herausforderungen begegnen können, die ich hier zusammenfassen möchte:

1. Technologiegestaltungsprozesse anzustoßen, welche die bestehenden Geschlechterordnung mit ihrer zweigeschlechtlich-heteronormativen Basis eher beugen (,diffract") als diese fortzusetzen,

2. nicht nur Produkte der Informatik im engeren Sinne zu gestalten, d. h. Software und Informations- und Kommunikationstechnologien, sondern auch alternative Gestaltung von Epistem(onto)logien der Modellierung und Algorithmen vorzuschlagen. Und

3. durch den Technikgestaltungsprozess einen Ort $\mathrm{zu}$ schaffen für Unerfassbares und „un/an/geeignete Andere“, der nach Haraway aus Interferenzmustern zusammengesetzt ist.

Nach den bisherigen Ausführungen erscheint ein solches Diffractive Design sehr gut geeignet für die die Informatik konstituierenden Aufgaben: die Modellierung von Wissen und die Konstruktion von Artefakten. Ein konsequentes interferentes Durch-einander-hindurch-Lesen von kritischen Technikgestaltungsansätzen mit Barad, Bohr und Haraway sowie Geschlechterforschung, feministischer „Epistem-onto-logie“ (Barad 2007, 2012) und Ethik bergen das Potential, im konkreten Fall informatische Artefakte auf der Basis sorgfältiger Analysen zu entwerfen und herzustellen, die sich auf traditionelle Objektivitätsverständnisse beziehen und diese zugleich geschlechterkritisch reformulieren. Ziel ist angesichts von Modernisierungsund Neoliberalisierungsstrategien die Konstruktion ,lebbare Welten“ im Sinne Haraways, die den „un/an/geeigneten Anderen“ einen Ort verschaffen, der ihnen/uns ein „lebenswertes Leben“ im Sinne Butlers ermöglicht. Diffractive Design ist die Vision einer verantwortungsvollen und feministischen Technikgestaltung, die eine radikal interdisziplinäre Zusammenarbeit von Geschlechterforscher_innen mit Technikgestalter_innen erforderlich macht.

\section{Literatur}

Agre, P. (1997). Computation and Human Experience. Cambridge: Cambridge University Press.

Akrich, M. (1995). User Representations: Practices, Methods and Sociology. In A. Rip, T. Misa \& J. Schot (Hrsg.), Managing Technology in Society (S. 167-184). London/New York: Pinter.

Allhutter, D. (2012). Mind Scripting: A Method for Deconstructive Design. Science, Technology \& Human Values, 37(6), 684-707.

Allhutter, D., Hanappi-Egger, E. \& John, S. (2008). Mind Scripting: Zur Sichtbarmachung von impliziten Geschlechtereinschreibungen in technologischen Entwicklungsprozessen. In B. Schwarze, M.

David \& B. Ch. Belker (Hrsg.), Gender und Diversity in den Ingenieurwissenschaften und der Informatik (S. 153-165). Bielefeld: Webler.

Barad, K. (2007). Meeting the Universe Halfway. Quantum Physics and the Entanglement of Matter and Meaning. Durham/London: Duke University Press.

Barad, K. (2012). Agentieller Realismus. Frankfurt a.M.: Suhrkamp. 
Barad, K. (2013). Diffraktionen. Differenzen, Kontingenzen und Verschränkungen von Gewicht. In C. Bath, H. Meißner, S. Trinkaus \& S. Völker (Hrsg.), Geschlechter Interferenzen. WissensformenSubjektivierungsweisen - Materialisierungen (S. 27-67). Münster [u. a.]: LIT Verlag.

Bath, C. (2007). „Discover Gender“ in Forschung und Technologieentwicklung? Soziale Technik, Zeitschrift für sozial- und umweltverträgliche Technikgestaltung, 4, 3-5.

Bath, C. (2009). De-Gendering informatischer Artefakte. Grundlagen einer kritisch-feministischen Technikgestaltung (Dissertation). Bremen: Staats- und Universitätsbibliothek Bremen. URN: http://nbn-resolving.de/urn:nbn:de:gbv:46-00102741-12

Bath, C. (2013). Semantic Web und Linked Open Data. Von der Analyse technischer Entwicklungen zum „Diffractive Design“. In C. Bath, H. Meißner, S. Trinkaus \& S. Völker (Hrsg.), Geschlechter Interferenzen. Wissensformen - Subjektivierungsweisen - Materialisierungen (S. 69-116). Münster [u. a.]: LIT Verlag.

Bath, C. (2014). Searching for methodology. Feminist technology design in computer science. In Waltraud Ernst \& Ilona Horwath (Hrsg.), Gender in Science and Technology. Interdisciplinary Approaches (S. 57-78). Bielefeld: transcript.

Bath, C. \& Both, G. (2014). Gendered Innovations - eine ambivalente Intervention in die Naturwissenschaften, Ingenieurwissenschaften und Medizin. Vortrag auf der Jahrestagung der Fachgesellschaft Gender Studies am 14./15.2.2014 in Paderborn.

Bath, C., Meißner, H., Trinkaus, S. \& Völker, S. (2013). Geschlechter Interferenzen. WissensformenSubjektivierungsweisen - Materialisierungen. Münster: LIT-Verlag.

Bauer, R. \& Götschel, H. (Hrsg.) (2006). Gender in den Naturwissenschaften. Ein Curriculum an der Schnittstelle der Wissenschaftskulturen. Talheim/ Mössingen: Talheimer.

Becker-Schmidt, R. \& Knapp, G. (Hrsg.) (2000): Feministische Theorien zur Einführung. Hamburg: Junius.

Bødker, S., Kensing, F., Simonsen, J. (2004). Participatory IT Design: Designing for Business and Workplace Realities. Cambridge: MIT Press.

Butler, J. (1991). Das Unbehagen der Geschlechter. Frankfurt a.M.: Suhrkamp. (Im Orig.: Butler, J. (1990). Gender trouble. Feminism and the subversion of identity. New York: Routledge.)

Cassell, J. (2003). Genderizing HCI. In J. Jacko \& A. Sears (Hrsg.), Handbook of Human-Computer Interaction (S. 402-411). Mahwah, NJ: Lawrence Erlbaum.

Degele, N. (2008). Gender/Queer Studies: Eine Einführung. Paderborn [u. a.]: UTB.

Flanagan, M., Nissenbaum, H., Diamond, J. \& Belman, J. (2007). A Method for Discovering Values in Digital Games. Situated Play. DiGRA'07 (Tokyo, JP, September 24-28, 2007). Verfügbar unter: http://valuesatplay.org/?page_id=12.

Friedman, B. \& Kahn, P. (2003). Human Values, Ethics, and Design. In J. Jacko \& A. Sears (Hrsg.), The human-computer interaction handbook (S. 1177-1199). Mahwah, NJ: Lawrence Erlbaum Associates.

Greenbaum, J. \& Kyng, M. (Hrsg.) (1991). Design at Work. Cooperative Design of Computer Systems. Hillsdale, NJ: Lawrence Erlbaum.

Hammel, M. (2003). Partizipative Softwareentwicklung im Kontext der Geschlechterhierarchie. Frankfurt a.M.: Peter Lang

Haraway, D. (1995). Monströse Versprechen. Eine Erneuerungspolitik für un/an/geeignete Andere. In D. Haraway, Monströse Versprechen. Coyote-Geschichten zu Feminismus und Technowissenschaft (S. 11-80). Hamburg: Argument. (Im Orig.: Haraway, D. (1992). Promises of the Monsters: A Regen- 
erative Politics for Inappropriate/d Others. In L. Grossberg, C. Nelson \& P. A. Treichler (Hrsg.), Cultural Studies (S. 295-337). New York/London: Routledge.)

Haraway, D. (1997).Modest_Witness@Second_Millennium.FemaleMale@_Meets_OncoMouse ${ }^{\mathrm{TM}}$. New York/London: Routledge.

Koreuber, M. (Hrsg.) (2010). Geschlechterforschung in Mathematik und Informatik. Baden-Baden: Nomos.

Kuhn, S. \& Muller, M. (Hrsg.) (1993). Special Issue on Participatory Design. Communications of the $A C M, 36(4)$.

Leicht-Scholten, C., (Hrsg.) (2007). Gender and Science. Perspektiven in den Natur- und Ingenieurwissenschaften. Bielefeld: transcript.

Maass, S. \& Wiesner, H., (2006). Programmieren, Mathe und ein bisschen Hardware ... Wen lockt dies Bild der Informatik? Informatik-Spektrum, 29(2), S. 125-132.

Quaiser-Pohl, C. \& Endepohls-Ulpe, M. (Hrsg.) (2010). Bildungsprozesse im MINT-Bereich: Interesse, Partizipation und Leistungen von Mädchen und Jungen. Münster: Waxmann.

Rommes, E., Bath, C. \& Maass, S. (2012): Methods for Intervention: Gender Analysis and Feminist Design of ICT. Science Technology Human Values, 37(6), S. 653-662.

Sengers, P., Boehner, K., David, S. \& Kaye, J. (2005). Reflective Design. In O. W. Bertelsen, N. O. Bouvin, P. G. Krogh \& M. Kyng (Hrsg.), Critical Computing - Between Sense and Sensibility (S. 4958). Red Hook, N.Y: Curran.

Siefkes, D., Eulenhöfer, P., Stach, H. \& Städtler, K. (1998). Sozialgeschichte der Informatik. Kulturelle Praktiken und Orientierungen. Wiesbaden: Deutscher Universitätsverlag.

Sørensen, K., Faulkner, W. \& Rommes, E. (2012). Technologies of Inclusion: Gender in the Information Society. Tapir Academic Press.

Stöger, H., Ziegler, A. \& Heilemann, M. (Hrsg.) (2012). Mädchen und Frauen in MINT: Bedingungen von Geschlechtsunterschieden und Interventionsmöglichkeiten. Münster: LIT.

Suchman, L. (2007). Human-Machine Reconfigurations. Plans and Situated Action (2. Auflage). Cambridge: Cambridge University Press.

Wagner, I., Brattetei, T. \& Stuehdahl, D. (2010). Exploring Digital Design: Multi-Disciplinary Design Practices. Berlin: Springer.

Wajda, K., Draude, C., Maass, S. \& Schirmer, C. (2013). GERD - Wo Gender, Diversity und Informatik zusammentreffen. In S. Boll, S. Maass \& R. Malaka (Hrsg.) (2013), Mensch \& Computer 2013 (S. 301-304). München: Oldenbourg Verlag.

Zorn, I., Maaß, S., Rommes, E., Schirmer, C. \& Schelhowe, H. (Hrsg.) (2007). Gender Designs IT. Construction and Deconstruction of Information Society Technology. Wiesbaden: Verlag für Sozialwissenschaften. 